Voix et Images

voixetimages

\title{
Éditer, creuser, critiquer
}

\section{Jacques Allard}

Volume 16, numéro 2 (47), hiver 1991

Jovette Marchessault

URI : https://id.erudit.org/iderudit/200905ar

DOI : https://doi.org/10.7202/200905ar

Aller au sommaire du numéro

Éditeur(s)

Université du Québec à Montréal

ISSN

0318-9201 (imprimé)

1705-933X (numérique)

Découvrir la revue

Citer cet article

Allard, J. (1991). Éditer, creuser, critiquer. Voix et Images, 16(2), 332-336.

https://doi.org/10.7202/200905ar

Ce document est protégé par la loi sur le droit d'auteur. L'utilisation des services d'Érudit (y compris la reproduction) est assujettie à sa politique d'utilisation que vous pouvez consulter en ligne.

https://apropos.erudit.org/fr/usagers/politique-dutilisation/
Cet article est diffusé et préservé par Érudit.

Érudit est un consortium interuniversitaire sans but lucratif composé de l’Université de Montréal, l'Université Laval et l'Université du Québec à Montréal. Il a pour mission la promotion et la valorisation de la recherche. https://www.erudit.org/fr/ 


\section{Recherches}

\section{Éditer, creuser, critiquer}

\section{par Jacques Allard, Université du Québec à Montréal}

Sur ma table se trouvent plusieurs ouvrages dont la plupart mériteraient bien une chronique entière. Sans être tous de la plus brûlante actualité, ils me paraissent pourtant bien utiles, parfois indispensables. Voici donc quelques fiches expresses. D'abord sur les publications de centres ou unités universitaires; puis sur trois numéros récents de revues. On constatera de nouveau la vigueur (maintenue depuis une bonne dizaine d'années) du travail historien et sociologique. On saluera le forum international qui souvent s'aménage en Europe, particulièrement en Italie.

Groupe de recherche sur l'édition littéraire au Québec (G.R.E.L.Q.), l'Édition du livre populaire (études sur les édi- 
tions Édouard Garand, de l'Étoile, Marquis, Granger Frères, rassemblées et présentées par Jacques Michon, Sherbrooke, Ex libris, 1988, 204 p. (Études sur l'édition); l'Édition de poésie (les éditions Erta, Orphée, Nocturne, Quartz, Atys et l'Hexagone), interview et études rassemblées et présentées par Richard Giguère, avec la collaboration d'André Marquis, Ex libris, 1989, 259 p. (Études sur l'édition).

Le G.R.É.L.Q, fondé en 1982 à l'université de Sherbrooke, n'a pas chômé. Après l'Édition littéraire au Québec de 1940 à 1960 (1985), sont venus ces deux titres. Nos abonné(e)s le savent un peu qui connaissent le dossier préparé pour nous l'an dernier par Jacques Michon (*L'édition littéraire au Québec*, Voix et images, $\mathrm{n}^{\circ} 41$, hiver 1989). Dans sa présentation de l'Édition du livre populaire, le collègue dit l'impossibilité de donner le tableau complet de l'activité des éditeurs populaires. Mais le menu offert est tout de même consistant, vu l'ignorance où nous en sommes généralement de l'ampleur et de la diversité du phénomène depuis le début du siècle.

Nostalgie garantie pour les plus vieux devant ces titres (et parfois les couvertures reproduites) de Granger et Frères ou Marquis: la sémiotique d'une époque s'y trahit. Mais surtout: de la belle et bonne ouvrage avec des illustrations (indétachables du genre), une table des références, les publications des chercheurs du G.R.É.L.Q., un index nominum et une table analytique des matières. En prime: une description signalétique de l'ouvrage qui mentionne non seulement le tirage (300, amateurs ne traînez pas) mais les marques du papier et de l'encre... Voilà des chercheurs de l'édition qui prennent date avec ceux du futur désencré et papyromane! Déjà on nous annonce d'ailleurs qu'au terme de ces recherches viendra une histoire de l'édition littéraire au Québec et au Canada français. L'introduction de Jacques Michon apporte là-dessus de premiers éléments très instructifs. Exemple: [au début du siècle] les écrivains ne sont pas assez nombreux pour alimenter les nouvelles machines à imprimer, Louvigny de Montigny disant: nous manquons de littérateurs populaires: s'entend d'écrivains qui se sont donnés la mission d'instruire le peuple en lui fournissant des livres à sa portée (p. 13).

Dans l'́dition de poésie, Richard Giguère respecte le modèle éditorial premier si ce n'est qu'il propose une entrevue avec Alain Horic (directeur des éditions de l'Hexagone) en remplacement de l'introduction attendue. Si les questions sont bonnes, les réponses laisseront les historiens sur leur appétit. Mais pour la petite (?) histoire de la vente récente de l'Hexagone au groupe « populaire " Sogides, il ne sera pas vain de s'y reporter. Et on en profitera pour mettre en rapport cette entrevue inauguratrice avec la fin du dossier consacré à la réception critique de l'Hexagone dans les revues, entre 1954 et 1970 . Toute la publication est d'ailleurs attentive au sens tant historique qu'idéologique ou esthétique de l'entreprise éditoriale. On y trouve aussi beaucoup d'illustrations de 
pages couvertures (là aussi le paratexte est parlant, autant que sur les couvertures populaires). Voilà un autre ouvrage de fond qui consacre le G.R.É.L.Q. comme l'un des centres outilleurs de nos recherches.

I.S. MacLaren et C. Potvin, Questions of funding, publishing and distribution/Questions d'édition et de diffusion (actes du deuxième colloque "Vers une histoire de l'institution littéraire au Canada»), Edmonton, University of Alberta, The Research Institute for Comparative Literature, 1989, 167 p.

C'est dans cet ouvrage (un peu mince, étant donné l'ampleur de l'entreprise) que l'on trouve une mise au point sur l'orientation des travaux dirigés par MM. Michon et Giguère. En particulier sur la périodisation de leur travail: ils partent des années 1920, puisque c'est à cette époque qu'apparaît pour la première fois dans notre histoire l'éditeur indépendant, non inféodé au clergé, à un journal ou à l'édition scolaire (p. 28).

L'ensemble de cette publication, comme ses titre et sous-titre l'annoncent, porte évidemment sur la problématique éditoriale pancanadienne. En ce qui concerne notre domaine, on se reportera aux contributions d'André Vanasse (sur le marché du livre au Québec et au Canada), de Sylvie Bernier (sur la fonction de l'illustration dans la diffusion et la légitimation), de Naïm Kattan (sur le rôle des gouvernements dans le financement), de Sherry Simon (sur le féminisme et les périodiques littéraires au Québec), de Jacques Pelletier (sur l'édition des Cahiers du département d'études littéraires de l'Université du Québec à Montréal). En postface où elle résume les propositions générales du colloque, Claudine Potvin rappelle aussi la position de son groupe de l'Université de l'Alberta sur le champ littéraire: on y a adopté l'approche polysystémique, sociologique, du littéraire selon laquelle il n'y a pas de littérature haute et basse, bonne et mauvaise. L'analyse de l'institution (règles, code, fonctions des appareils) suppose l'examen de tous les éléments, de toutes les voix ou langages (interreliés, interagissants ou réagissañts) du territoire dit littéraire. Prochains colloques annoncés par le groupe: les préfaces et manifestes; les littératures de moindre diffusion; les genres littéraires; la littérature au féminin; les traditions orales indigènes; la traduction littéraire; la paralittérature; la canonisation (sélection, valorisation et consécration des œuvres littéraires). Avis aux intéressé(e)s.

Franca Marcato Falzoni, La deriva delle francofonie, atti dei seminari annuali di Letterature Francofone. Autour de l'univers souterrain dans la littérature québécoise, Bologne, Cooperativa Libraria Universitaria Editrice Bologna (CLUEB, Via Marsala 24, 40126 Bologna), 265 p. (Bussola, n 7 ).

Treize interventions qui creusent (certains piochent, d'autres fouissent) le texte national. Patrick Imbert, * Les souterrains idéolo- 
giques»; Naïm Kattan, «La réconciliation dans la littérature québécoise "; Francesca Morale, "Ambiguité dans le genre narratif "terrifiant" du XIX" siècle québécois "; Jean Larose, "De quelques vers en germe chez Octave Crémazie "; Marco Modenesi, "Eaux de surface et eaux de profondeur dans la poésie de Grandbois"; Robert Schwartzwald, "Dresser le souterrain: réhabilitation et refoulement de l'automatisme à la première Rencontre des écrivains canadiens "; Marie Lyne Piccione, "Le diable et sés avatars dans le roman québécois contemporain "; Carla Fratta, «Le mal des cloîtres. Espace souterrain et univers clos dans l'œuvre romanesque d'Anne Hébert"; Jean-Marcel Paquette, «Paradoxe de l'univers infernal chez Jacques Ferron "; Liana Nissim, *Roland Giguère: la stratification souterraine de l'univers ou la cosmographie de la nuit»; Jósef Kwaterko, "Le souterrain "autorisé" chez Jacques Godbout"; Madeleine Frédéric, "La mort et ses transfigurations de Jean le Maigre à Judith Lange"; Lise Gauvin, "L'en-deça des voyages: exploration de quelques romans québécois". Avec une "Bibliographie" (ouvrages cités) par Paola Puccini et un "Index des noms * par Alessandra Ferraro: À remarquer dans cette autre initiative du dynamique * Centro di letteratura quebeccese" de Bologne: la réunion périodique d'une assemblée internationale sur notre littérature; cette fois, à côté des Québécois se sont retrouvés des Français, Belge, États-Unien et Polonais bien connus.

Littératures, Cahiers du département de langue et littératures françaises, Faculté des Lettres, université McGill, Montréal, $n^{\text {os }} 1$ et 2, 1988, 199 et 154 p. (sous la direction d'Yvan Lamonde ${ }^{1}$ ).

De leur côté, ces articles vont au cœur (souvent ici très riche) de l'histoire. Au sommaire du premier numéro: Yvan Lamonde, "Institution et associations littéraires au Québec au XIXe siècle: le cas de l'Institut Canadien de Montréal "; Marc Angenot, "Le roman français dans la bibliothèque de l'Institut canadien de Montréal (1845-1876)»; Jane Everett, "Orthodoxie et hétérodoxie littéraires: le cas du Québec vers 1900"; Élisabeth Nardout-Lafarge, "Autonomie littéraire et rupture symbolique: le Québec et la France, 1940-1950 "; Jean-Pierre Boucher, "Point de vue narratif dans Alexandre Chenevert ". Avec un * Document: thèses de doctorat et mémoires de maîtrise au Département de langue et littérature françaises de McGill (1906-1987) „: 78 sujets « québécois" sur 592.

Le deuxième numéro comprend les articles d'André Smith: * La circulation du pouvoir dans Tit-Coq et Bousille et les justes"; Marcel Fortin: "La réception critique de l'œuvre d'Anne 'Hébert, l'histoire d'une célébration *; Jean-Pierre Boucher, "Jacques Ferron et le recueil: la Conférence inachevée ". On y trouve aussi, comme "Documents", deux lettres de Ferron et un index des auteurs étudiés dans les thèses et mémoires de McGill. 
Je termine avec trois mentions de revues que réunit une préoccupation critique. Le dernier numéro des Écrits du Canada français (70, octobre 1990) donne les actes du colloque * La critique face à ellemême * sous le titre * Critique(s)/Écrivains/Lecteurs *. Intervenants : J.-P. Duquette, J.-G. Pilon, J. Éthier-Blais, J. Allard, A. Brochu, M. Ouellette-Michalska, S. Simon, P.-A. Bourque, R. Lapierre, G.-P. Ouellette, G. Cloutier, P. Nepveu, J. Royer, G. Toupin, R. Martel, B. Roy et M. Roy.

Le premier numéro de la Revue internationale d'études canadiennes $\left(\mathrm{n}^{\circ} 1\right.$, printemps-automne 1990, 2, avenue Daly, Ottawa, K1N 6E2) est consacré à * La recherche sur le Canada * et comprend une contribution de votre serviteur: «Où en sont les études sur la littérature québécoise?» (p. 114-134). Enfin, la dernière livraison de Canadian Literature (Littérature canadienne) comporte un article d'Agnès Whitfield: "Gabrielle Roy as Feminist: Re-reading the Critical Myths * (p. 20-31).

Critiquer? creuser? éditer? Ce sera toujours d'abord affaire de lecture. Voyez par vous-même!

1 Ma chronique précédente ( $\AA$ quiand une histoire de la pensée au Québec») m'a valu du courrier. D'abord de Yvan Lamonde, auteur de la bibliographie dont j'ai parle (L'histoire des idées au Quebec, 1760-1960: bibliographie des études). L'espace de cette chronique étant insuffisant pour faire vraiment place au débat, Yvan Lamonde a été invité à préparer un article à paraître en nos pages plus tard. De son côté, M. Robert Hébert m'a signalé son article portant sur le thème même d'une pensée nationale, laquelle ne parait pas exister pour les philosophes d'ici à moins qu'ils ne parlent de la pensée allemande, française, danoise ou autre. Voir «Fracture endo-coloniale: autour d'un anniversaire et de quelques identités ", la Petite Revue de philosophie, vol. XI, n 1 , automne 1989, p. 63-83. 